\title{
Littéracies universitaires : accompagnement et autonomisation dans l'apprentissage des écrits de et à l'université
}

Sophie Bailly and Véronique Lemoine-Bresson

\section{(2) OpenEdition \\ Journals}

Electronic version

URL: http://journals.openedition.org/ripes/2608

DOI: 10.4000/ripes.2608

ISSN: 2076-8427

Publisher

Association internationale de pédagogie universitaire

\section{Electronic reference}

Sophie Bailly and Véronique Lemoine-Bresson, "Littéracies universitaires : accompagnement et autonomisation dans l'apprentissage des écrits de et à l'université", Revue internationale de pédagogie de l'enseignement supérieur [Online], 36(2) | 2020, Online since 19 September 2020, connection on 29 January 2021. URL: http://journals.openedition.org/ripes/2608 ; DOI: https://doi.org/10.4000/ripes. 2608

This text was automatically generated on 29 January 2021.

Article L.111-1 du Code de la propriété intellectuelle. 


\title{
Littéracies universitaires : accompagnement et autonomisation dans l'apprentissage des écrits de et à l'université
}

\author{
Sophie Bailly and Véronique Lemoine-Bresson
}

1 Ce numéro thématique est issu du croisement de deux projets. Le premier projet était consacré aux littéracies universitaires dans une collaboration scientifique d'envergure entre des chercheurs de trois universités françaises et quatre brésiliennes (CapesCOFECUB SH 834-15 2015-2018; Bailly et al., 2016a). Le second projet touche à la pédagogie universitaire et plus précisément à la préoccupation enseignante de mettre en place des dispositifs pour que tous les étudiants réussissent leurs apprentissages de l'écriture universitaire. La question des littéracies universitaires, dont l'orthographe varie selon le contexte de production, préoccupe les chercheurs depuis plus de trente ans (Hayes et Flower, 1986) et s'installe dans le monde francophone et lusophone avec les travaux de Rinck et al. (2015). Des chercheurs comme Boch et Grossman (2001), Rinck (2006) ou plus récemment Lopes Dias Ignácio Rodrigues (2016) ont largement décrit la question de l'identité de l'auteur ainsi que les processus et les composantes qui sont à l'œuvre dans les écrits à l'université, comme l'argumentation, la polyphonie, la pratique de la citation. Associées à la problématique de l'accompagnement et de l'autonomisation des étudiants dans le développement complexe de la lecture et l'écriture académique, les littéracies universitaires donnent des pistes intéressantes pour mettre en place des dispositifs d'enseignement pour tous. En effet, il s'agit d'une manière générale, et ce depuis plus de trois décennies (Loi Savary, n ${ }^{\circ} 84-52$ du 26 janvier 1984), d'éviter le décrochage des étudiants, par la mise en place d'accompagnements dont les modalités peuvent être diverses.

2 On observe que de nombreux travaux ont été consacrés à la description des normes de rédaction des genres qui circulent dans le monde universitaire et scientifique, ou à l'appropriation de ces normes par des étudiants. Les études qui interrogent les freins et 
les leviers aux capacités de lire et d'écrire, mais également de comprendre et de produire des textes aux normes différentes sont, certes, centrales pour aider les étudiants qui forment «un ensemble de moins en moins homogène " (Bailly et al., 2016b, p. 5), mais également cruciales pour tout enseignant à l'université quelle que soit sa discipline. Or, il existe peu de documentation scientifique sur les pratiques d'aide et d'accompagnement à l'apprentissage de la rédaction pour tous - étudiants et enseignants -, c'est-à-dire sur les modalités pédagogiques d'accompagnement à l'entrée en écriture, plutôt que sur l'enseignement des règles de l'écriture dont les limites sont aujourd'hui bien connues.

3 C'est pourquoi, nous avons souhaité appréhender la question de l'enseignement des littéracies universitaires à partir des modalités d'aide et d'accompagnement qui privilégient l'organisation de dispositifs centrés sur l'autonomisation des apprenants ou sur le travail collectif et collaboratif. Les recherches présentées mettent au jour les paramètres à observer pour rendre dynamique l'apprentissage des étudiants. Il est question d'interroger le degré de guidage dans la réalisation d'une tâche et les phases de régulation, la vitesse d'écriture et de réécriture et ses effets sur les performances en écriture ou encore la gestion du temps. L'attention est portée sur le Learning Knowledge ou culture d'apprentissage, avec la définition des objectifs, liés au contenu, et sur la compétence d'apprentissage appelée «maîtrise stratégique » par Holec et al. (1996), les ressources ou les stratégies cognitives. Les dispositifs présentés s'intéressent particulièrement aux étudiants qui sont autorisés à prendre en charge leur travail, à choisir leurs sujets d'écriture, leurs ressources - parmi le matériel à disposition, leurs espaces de travail, leurs partenaires; ce qui favorise amplement le développement de l'autonomie selon Benson (2011). L'enjeu de ces dispositifs est de favoriser, dans l'acculturation aux écrits universitaires, "l'exercice actif de la responsabilité d'apprenant; la capacité d'apprendre est donc à développer pour pouvoir prendre en charge toutes les décisions inhérentes à l'apprentissage » (Chateau et al., 2015, parag. 5). Des facteurs autonomisants sont problématisés, comme l'implication de l'apprenant dans les différentes étapes du processus d'écriture, le choix des objectifs en fonction de son stade de développement de compétences ou les décisions d'autorégulation. La question des dispositifs pédagogiques peut également faire le jour sur le recours raisonné aux technologies numériques (Bailly, 2010) et sur le renouvellement de modes de relation, d'interaction et de médiation pédagogiques qui soient plus symétriques. Ce dernier point soulève la question des compétences relationnelles des enseignants, que ceux-ci développent entre eux quand le dispositif est codirigé ou négocié avec les étudiants. Toutes ces dimensions mettent en tension l'espace de la recherche et celui des pratiques pédagogiques à l'université, avec pour objectif, le rappelle Lison (2018), de «créer des connaissances afin de comprendre puis (peut-être) d'améliorer les pratiques de nos collègues [et les nôtres - nous ajoutons -], et ce faisant, favoriser l'apprentissage des étudiants » (p. 1). En effet, les dispositifs pédagogiques présentés et analysés montrent comment les enseignants apprennent de leurs erreurs et sont prêts à revisiter les modalités d'enseignement et de communication envers les étudiants.

Dans ce numéro thématique, nous proposons cinq contributions qui s'inscrivent dans une "pédagogie de la coopérativité » où le processus d'apprentissage de l'écriture universitaire est conçu comme un dialogue entre l'enseignant et l'étudiant (Barbot et Camatarri, 1999, p. 19). Pour toutes les contributrices, l'enjeu majeur est celui de la réussite de tous les étudiants dans leurs études supérieures. Une contribution vient de Suisse, l'autre de Tunisie, tandis que les trois suivantes viennent de France. Le profil 
pluridisciplinaire des autrices montre que la question intéresse tout enseignant soucieux de la réussite de tous les étudiants, quels que soient leurs profils : en Licence, en Master, en reprise d'études, en langue maternelle ou en langue étrangère. Les articles s'articulent autour des thématiques suivantes :

- objectifs, contenus, contextes, dispositifs et outils didactiques pour aider l'apprentissage des littéracies universitaires en anglais scientifique

-Évaluation et diagnostic de la compétence d'écriture chez des étudiants novices et des étudiants experts en FLE

- Évaluation, autoévaluation, co-évaluation des écrits universitaires : critères, outils, méthodes

- Pratiques de formation, de co-formation et autoformation aux écrits universitaires et scientifiques

- Innovations pédagogiques et technologiques pour la formation à lire et écrire des discours universitaires et scientifiques.

5 Le premier article est proposé par Nada Najjar, jeune enseignante-chercheuse en psychologie à la Faculté des Sciences Humaines et Sociales à Tunis. Elle a en charge 32 étudiants de divers profils: des adultes en reprise d'étude, des étudiants redoublants et des étudiants nouveaux entrants. La particularité de ces étudiants est de devoir apprendre l'écriture universitaire en français, langue considérée dans le contexte en tant que langue étrangère (FLE), car cette compétence conditionne grandement leur réussite aux examens. Dans son dispositif, Najjar s'intéresse à la tâche rédactionnelle tapuscrite enregistrée via un logiciel de capture vidéo. L'analyse de son dispositif montre qu'il existe une forte dépendance entre les stratégies que les étudiants mobilisent pour réguler leur rédaction et leurs performances à l'écrit, mais également que ces stratégies ont un lien avec l'expérience d'écriture préalable.

6 Le deuxième article s'inscrit également dans le champ de l'écriture en langue étrangère à l'université. Sophie Bailly s'intéresse depuis de nombreuses années aux hétérogénéités linguistiques et langagières des étudiants dans le cours d'anglais scientifique du master didactique des langues/fle de l'université de Lorraine. Dans cet article, elle veut se distancier de son dispositif en vue de l'améliorer. C'est pourquoi elle a sollicité un regard extérieur d'une collègue, Véronique Lemoine-Bresson, pour coanalyser le design du système d'aide proposé. Les résultats montrent que les étudiants trouvent des intérêts particuliers dans le dispositif quant au développement de leur autonomie en tant que moteur pour mieux apprendre à écrire en anglais. En même temps, ils soulignent la complexité de la tâche d'écriture. Soucieuse de l'implication des étudiants et de l'accrochage à la tâche demandée, Sophie Bailly peaufine les ingrédients importants de l'autorégulation et les interactions directes avec les étudiants par le biais d'entretiens conseils et d'un journal de bord.

Le troisième article, rédigé par Marlène Lebrun, spécialisée en langue et littérature françaises, de la Haute École Pédagogique de Béjune en Suisse, met des outils de réflexivité au centre du dispositif pédagogique proposé : à savoir le journal de lecture dialogué ou cahier culturel partagé et le cahier d'écrivain. La parole est donnée à des étudiants en formation à l'enseignement primaire ou secondaire pour qu'ils expriment leur point de vue sur ces outils que Lebrun a modélisés et fait évoluer depuis 2010. Les étudiants soulignent les effets sur l'empathie et la distanciation qu'ils développent dans 
un espace défini comme un lieu d'expérimentation des fonctions cognitive, réflexive et heuristique de l'écriture.

Une équipe pluridisciplinaire de cinq chercheurs de l'université de Lorraine signent le quatrième article. Une chercheuse en Inspé (Martine Paindorge), deux en Faculté des Sciences et Technologies Virginie Libante et Bertrand Aigle), un en Faculté de médecine (Pascal Reboul) et une à la Bibliothèque Universitaire des Sciences et Techniques (Virginie Lang) ont mis en place un dispositif dans le cadre du Cursus Master Ingénieur (CMI) de Biologie Santé Environnement (BSE). Il concerne des étudiants de première année de cette formation et propose une Activité de Mise en Situation (AMS) qui contribue à autonomiser des étudiants tout juste sortis du lycée. L'originalité de la contribution vient des fortes dimensions coopératives, tant dans l'équipe des cinq chercheurs que dans le groupe d'étudiants.

Le cinquième article est écrit par Claire Polo, enseignante-chercheuse en sciences de l'éducation et de la formation, qui présente le dispositif Créer Lire Ecrire et Faire de la Science pour l'Université (CLEFS), expérimenté depuis novembre 2018 à l'université Lyon 2. Les conditions de travail sont exceptionnelles et qualitatives. Un accompagnement dit d'accrochage aux études supérieures est prévu pour des étudiants de première année de licence. L'approche pédagogique s'inspire des principes de l'Affective Learning Together. Elle veut tenir à distance le modèle déficitaire de réparation de lacunes supposées. Elle valorise plutôt les ressources des personnes volontairement engagées dans le dispositif, qui pour le moment en est à sa phase d'expérimentation.

\section{BIBLIOGRAPHY}

Bailly, S. (2010). Supporting Autonomy Development in Online Learning Environments: What Knowledge and Skills do Teachers Need? Dans M. J. Luzon, M. N. Ruiz et M. L. Villanueva (dir.), Genres Theory and New Literacies: Applications to Autonomous Language Learning (p. 81-100). Cambridge Scholars Publishing

Bailly, S, Alves Assis, J. et Deneire, M. (2016a). Écrire et apprendre à écrire à l'université brésilienne, française et québécoise : questions de recherche en didactique des langues. Mélanges CRAPEL, (37/1).

Bailly, S, Alves Assis, J. et Deneire, M. (2016b). Introduction. Mélanges CRAPEL, (37), 5-9.

Barbot, M. J. et Camatarri, G. (1999). Autonomie et apprentissage. PUF.

Benson, P. (2011). Teaching and researching autonomy. Pearson.

Boch, F. et Grossmann, F. (2001) De l'usage des citations dans le discours théorique. Des constats aux propositions didactiques. Lidil, 24, 91-111.

Chateau, A., Bailly, S. et Ciekanski, M. (2015). Vers l'institutionnalisation de l'enseignement autonomisant - La technologie, soutien et obstacle à l'innovation. Alsic. https://doi.org/10.4000/ alsic.2838. 
Hayes, J. R. et Flower, L. S. (1986). Writing research and the writer. American Psychologist, 41, 1106-1113.

Holec, H., Little, D. et Richterich, R. (1996). Stratégies dans l'apprentissage et l'usage des langues. Conseil de l'Europe.

Lison, C. (2018). La pédagogie de l'enseignement supérieur : d'une science à des pratiques diversifiées. Ripes, 34(1), 1-3. https://journals.openedition.org/ripes/1295

Loi Savary, n84-52 du 26 janvier 1984 sur l'enseignement supérieur, J.O. du 22 juin 2000. https:// www.legifrance.gouv.fr/affichTexte.do?cidTexte=JORFTEXT000000692733

Lopes Dias Ignácio Rodrigues, D. (2016). Discurso relatado : entre semelhança e diferença. Mélanges CRAPEL, (37/1), 119-128.

Rinck, F. (2006). Écrire au nom de la science et de sa discipline : les figures de l'auteur dans l'article en sciences humaines. Sciences de la Société, 67, 95-112.

Rinck, F., Boch, F. et Alves Assis, J. (2015). Letramento e formação universitariá : formar para a escrita. Mercato de Letras.

\section{AUTHORS}

\section{SOPHIE BAILLY}

Equipe Didactique des langues et sociolinguistique, Université de Lorraine, Coordinatrice du numéro thématique

\section{VÉRONIQUE LEMOINE-BRESSON}

Equipe Didactique des langues et sociolinguistique, Université de Lorraine, Coordinatrice du numéro thématique 